\title{
Successful closure of a gastropulmonary fistula after esophagectomy using the Apollo Overstitch and endoscopic vacuum therapy
}

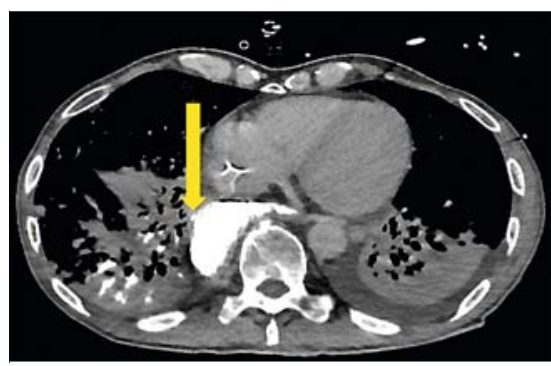

- Fig. 1 Contrast-enhanced axial computed tomography (CE-CT) scan showing a leak from the pull-up gastric conduit interponate to the right lung parenchyma (yellow arrow).

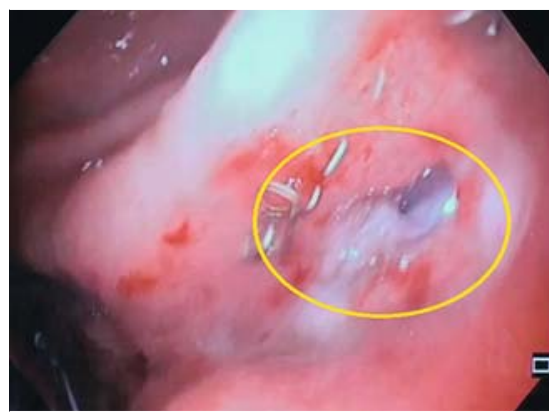

$\checkmark$ Fig. 2 Endoscopic view showing the gastropulmonary fistula (yellow circle) along the longitudinal suture line of the pull-up gastric interponate.

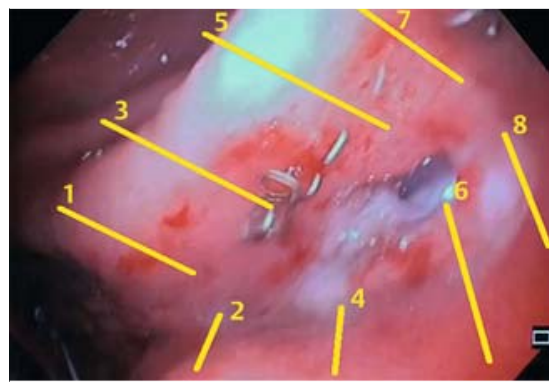

- Fig. 3 The suturing pattern for the four parallel stiches (yellow) used to perform the single-suturing technique. Closure was obtained by positioning the sutures as following: (1) oral anterior wall; (2) aboral anterior wall; (3) oral anterior wall; (4) aboral anterior wall; (5) oral anterior wall; (6) aboral anterior wall; (7) aboral anterior wall; and (8) oral anterior wall.

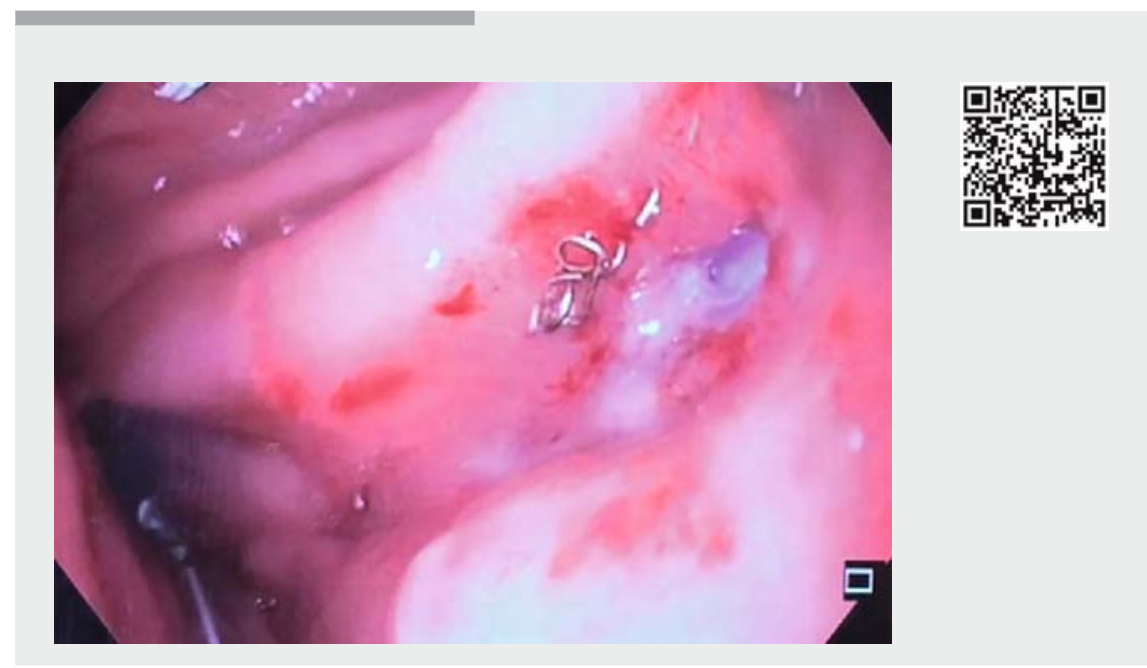

Video 1 Successful closure of a gastropulmonary fistula after Ivor Lewis esophagectomy using a combination of endoscopic suturing and endoscopic vacuum therapy.

Gastropulmonary fistulas following transthoracic en bloc esophagectomy and intrathoracic gastric reconstruction (Ivor Lewis esophagectomy) are rare but life-threatening complications. Surgical management of these is difficult and associated with significant instances of morbidity and mortality [1]. One possible treatment for leaks after upper gastrointestinal surgery is endoluminal vacuum therapy (EVT) [2]. A newer treatment option is the endoscopic suturing system Apollo Overstitch [3-5]. The Overstitch is a single-use device that is placed on top of a double-channel therapeutic endoscope, which facilitates full-thickness surgical suturing in single or running technique. To our knowledge, the case presented below constitutes the first successful clinical application of a combination of Overstitch and EVT to close a gastropulmonary fistula after Ivor Lewis esophagectomy.

We present the case of a 58 -year-old man who underwent neoadjuvant chemoradiation (CROSS protocol) followed by Ivor Lewis esophagectomy for esoph- ageal adenocarcinoma (histopathology: ypT3, ypN2 (3/45), L1, V0, Pn0, R0). About 3 months after the initial surgery, the patient was admitted to our hospital with recurring episodes of pneumonia. Computed tomography (CT) scanning with oral contrast and endoscopy showed a fistula between the pull-up gastric interponate and the right lung parenchyma ( $\triangleright$ Fig. 1 and $\triangleright$ Fig. 2 ).

We applied the Overstitch system to close the fistula endoscopically. After two attempts, we were able to close the fistula using the single-suturing technique ( $\vee$ Fig. 3 ; $\triangleright$ Video 1). A triple-lumen diverted nasal tube (e.g. Freka Trelumina; Fresenius Kabi, Germany) was then inserted to allow the patient to receive enteral feeding. Furthermore, to evacuate any biliary reflux and to reduce the pressure on the suturing line, we placed a polyurethane foam drainage tube (e.g. EsoSponge; Braun, Germany) with negative pressure $(-125 \mathrm{mmHg}$, continuous; VivanoTec; Hartmann AG, Germany). During the 31 days of this EVT, the foam drainage was changed seven times. 
The patient's infection parameters decreased with this treatment, and a follow-up endoscopy (day 34 after surgery) showed successful closure of the fistula.

Endoscopy_UCTN_Code_TTT_1AO_2AI

Competing interests

None

The authors

Seung-Hun Chon ${ }^{1}$, Ulrich Toex ${ }^{2}$, Patrick Sven Plum $^{1}$, Claudia Fuchs ${ }^{1}$, Robert Kleinert ${ }^{1}$, Christiane Bruns ${ }^{1}$, Tobias Goeser ${ }^{2}$

1 Department of General, Visceral and Cancer Surgery, University Hospital of Cologne, Germany

2 Department of Gastroenterology and Hepatology, University Hospital of Cologne, Germany
Corresponding author

\section{Seung-Hun Chon, MD}

General, Visceral and Cancer Surgery, University Hospital of Cologne, Kerpener Str. 62, 50937 Cologne, Germany

Fax: +49-221-47886227

seung-hun.chon@uk-koeln.de

\section{References}

[1] Lambertz R, Hölscher AH, Bludau M et al. Management of tracheo- or bronchoesophageal fistula after Ivor-Lewis esophagectomy. World J Surg 2016; 40: 1680-1687

[2] Bludau M, Fuchs HF, Herbold T et al. Results of endoscopic vacuum-assisted closure device for treatment of upper Gl leaks. Surg Endosc. doi:10.1007/s00464-017-5883-4

[3] Stavropoulos SN, Modayil R, Friedel D. Current applications of endoscopic suturing. World J Gastrointest Endosc 2015; 7: 777 789

[4] Kantsevoy SV, Bitner M, Hajiyeva G et al. Endoscopic management of colonic perforations: clips versus suturing closure (with videos). Gastrointest Endosc 2016; 84: 487-493

[5] Belfiori V, Antonini F, Deminicis S et al. Successful closure of anastomotic dehiscence after colon-rectal cancer resection using the Apollo overstitch suturing system. Endoscopy $2017 ; 49$ : $823-824$

\section{Bibliography}

DOI https://doi.org/10.1055/a-0592-6384

Published online: 13.4.2018

Endoscopy 2018; 50: E149-E150

(c) Georg Thieme Verlag KG

Stuttgart · New York

ISSN 0013-726X

\section{ENDOSCOPY E-VIDEOS}

https://eref.thieme.de/e-videos

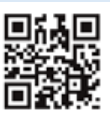

Endoscopy E-Videos is a free access online section, reporting on interesting cases and new

techniques in gastroenterological endoscopy. All papers include a high quality video and all contributions are freely accessible online.

This section has its own submission website at https://mc.manuscriptcentral.com/e-videos 